\title{
The Role of Prefrontal Cortex in Verbal Episodic Memory: rTMS Evidence
}

\author{
Marco Sandrini ${ }^{1}$, Stefano F. Cappa ${ }^{2}$, Simone Rossi ${ }^{3}$, \\ Paolo M. Rossini ${ }^{1,4}$, and Carlo Miniussi ${ }^{1}$
}

\begin{abstract}
Long-term, episodic memory processing is supposed to involve the prefrontal cortex asymmetrically. Here we investigate the role of the dorsolateral prefrontal cortex (DLPFC) in encoding and retrieval of semantically related or unrelated word pairs. Subjects were required to perform a task consisting of two parts: a study phase (encoding), in which word pairs were presented, and a test phase (retrieval), during which stimuli previously presented had to be recognized among other stimuli. Consistently with our previous findings
\end{abstract}

\section{INTRODUCTION}

The prefrontal cortex is not solely devoted to working memory processes (Goldman-Rakic, 1996; Petrides, 1994; Stuss, Eskes, \& Foster, 1994), but is also involved in long-term episodic learning (Fletcher \& Henson, 2001). The ability to consciously remember an experience requires its initial encoding, and its subsequent retrieval from long-term memory. Recent findings from functional imaging studies have provided evidence for hemispheric asymmetry in memory encoding and retrieval (HERA model). According to this model, the left prefrontal cortex is involved in the encoding of information about novel events into episodic memory and in the retrieval of information from semantic memory, whereas the right prefrontal cortex is involved in the retrieval of information from episodic memory (Tulving, Kapur, Craik, Moscovitch, \& Houle, 1994). In its original proposal, the HERA model was limited to verbal materials; the model was then extended to nonverbal materials by Nyberg, Cabeza, and Tulving (1996).

Because the HERA model is essentially based on neuroimaging studies, it should be validated using other sources of information. Neuroimaging results sometimes are not in agreement with lesion studies, and numerous different reasons may account for this discrepancy. Functional neuroimaging is helpful in identifying brain

\footnotetext{
${ }^{1}$ IRCCS S. Giovanni di Dio-FBF Brescia, ${ }^{2}$ Università Vita-Salute S. Raffaele, Milan, ${ }^{3}$ Policlinico Le Scotte, Sienna, ${ }^{4}$ Università Campus Biomedico Rome, Italy
}

using pictures, repetitive transcranial magnetic stimulation (rTMS) had a significant impact on episodic memory. The performance was significantly disrupted when rTMS was applied to the left or right DLPFC during encoding, and to the right DLPFC in retrieval, but only for unrelated word pairs. These results indicate that the nature of the material to be remembered interacts with the encoding-retrieval DLPFC asymmetry; moreover, the crucial role of DLPFC is evident only for novel stimuli.

regions involved in a given task but generally cannot distinguish between areas that are playing a critical role in it (Price \& Friston, 1999). In contrast, information gained from lesion studies sometimes demonstrates that the damage of a specific brain region, known to be active from brain imaging, does not lead to an inability to perform the same task. Although such results suggest that the area in question is not crucial for the task, their interpretation is complicated by the possibility that adaptive processes, such as diaschisis or plastic functional reorganization, have been induced by the lesion. The use of repetitive transcranial magnetic stimulation (rTMS) has the advantage of combining lesion and neuroimaging approaches, allowing to gain more information on "functionally relevant areas." RTMS is an excellent tool for the direct investigation of the functional participation of a brain area in an ongoing cognitive process (Walsh \& Cowey, 2000). Indeed, this approach does not depend on the measurement of the electrophysiological or hemodynamic responses to cognitive challenge; thus, it complements traditional neuroimaging techniques (PET; fMRI; ERP), in offering the unique opportunity to interact directly with the functioning of a cortical area and related neuronal circuitry, during the execution of a defined task. This can elucidate different stages of memory processes, like encoding and retrieval, indicating which areas are necessary or predominant for successful performance.

In a previous study from this laboratory (Rossi et al., 2001), rTMS was used to interfere transiently with either left or right prefrontal brain activity during encoding or 
retrieval of pictures showing complex scenes. Results indicated that in young adults the left dorsolateral prefrontal cortex (DLPFC) has an active role in encoding operations (with a functional prevalence into the right), whereas the right DLPFC is crucial for the retrieval of the encoded information, in agreement with the hemispheric encoding-retrieval asymmetry (HERA) model (Tulving et al., 1994).

The interaction of the nature of the material to be remembered with the hemispheric asymmetry predicted by the HERA model appears to be complex. Both the material and the type of memory process may affect the lateralization of frontal activation during memory tasks (Fletcher \& Henson, 2001).

A review of the literature has led Cabeza and Nyberg (2000) to conclude that in encoding of verbal materials, prefrontal activations are often left lateralized, whereas nonverbal stimuli sometimes yield bilateral and rightlateralized activation (Brewer, Zhao, Desmond, Glover, \& Gabrieli, 1998; Kelley et al., 1998; Wagner et al., 1998; Roland \& Gulyas, 1995). It is noteworthy that semantic memory retrieval tasks, which involve episodic memory encoding, consistently yield left-lateralized activation for both verbal and nonverbal materials (Gorno-Tempini et al., 1998; Thompson-Schill, D'Esposito, Aguirre, \& Farah, 1997; Vandenberghe, Price, Wise, Josephs, \& Frackowiak, 1996; Martin, Haxby, Lalonde, Wiggs, \& Ungerleider, 1995; Martin, Wiggs, Ungerleider, \& Haxby, 1996).

Regarding the retrieval conditions, it has been shown that prefrontal activation is sometimes bilateral, even if there is a clear tendency for right lateralization. The functional significance of right predominance during retrieval has been the subject of much debate. One proposal is that the right prefrontal activation reflects the adoption of a retrieval mode: the state arising whenever one refers back in time to past experiences (Kapur et al., 1995; Nyberg et al., 1995). An alternative hypothesis is that prefrontal activation reflects the degree of retrieval effort, the right (and left) prefrontal cortex being more active when retrieval is difficult (Schacter, Alpert, Savage, Rauch, \& Albert, 1996). A third proposal is that the right prefrontal activation reflects processes operating after retrieval of information form episodic memory. Such postretrieval processes might include the monitoring of whether the retrieved information is sufficient for the current task (Shallice et al., 1994) and the utilization of such information to guide behavior (Rugg, Fletcher, Frith, Frackowiak, \& Dolan, 1996).

The aim of the present study was twofold: first, to assess the effects of a change in stimulus material on the hemispheric asymmetry observed in the previous study and, second, to evaluate the effects of "stimulus novelty" on the same experimental paradigm. This stems from unpublished observations on the effect of rTMS on behavioral performance in the picture encoding/retrieval task, which showed that the interference effects could not be observed in a sample of subjects (involved in various phases of the experiment) who were familiar with the stimulus materials, suggesting that the DLPFC could be engaged in episodic memory processing only when novel material is processed. However, it was impossible in that group to disentangle the effects due to the task practice from effects due to the novelty of stimuli. Petersen, van Mier, Fiez, and Raichle (1998) reported progressively reduced frontal activation with practice increasing, suggesting a relation to the requirement of more elaborate information processing during encoding of novel information, as well as to the different retrieval demands of the nonpracticed task (Rugg \& Wilding, 2000).

For this reason, we used a task that assessed encoding and retrieval for familiar stimuli (semantically related word pairs) versus memory for novel stimuli (semantically unrelated word pairs). Specifically, in the present study, we applied the same methodology of the previous study to disrupt transiently the function of the left or right DLPFC to clarify their respective roles and functional hierarchy in the mechanisms of encoding and retrieval of semantically related or unrelated word pairs.

\section{RESULTS}

In the current experiment, participants were required to perform a long-term memory task consisting of two phases: a study phase (encoding), in which word pairs (semantically related or unrelated) were presented, and a test phase (retrieval), during which stimuli previously presented (tests) had to be recognized from among other stimuli (distractors). The test phase examined whether rTMS interference during encoding and retrieval was present for both types of word pairs, related or unrelated (see Figure 1). Overall, the participants' accuracy was very high for the relatedness task and the error rate was similar across all blocks (mean error rate $1.9 \%$ ), whereas in the recognition task the accuracy was strictly related to the rTMS manipulation (mean error rate $9.02 \%$ ).

Differences between category and blocks (rTMS stimulation conditions) emerged in retrieval: The accuracy was significantly higher for related word pairs than for unrelated word pairs, $F(3.1,34.3)=4.55, p<.05$, ANOVA using Huynh-Feldt epsilon correction when appropriate, indicating a different effect by rTMS between the two categories. We interpreted this to mean that with unrelated word pairs, memory processes at play in the DLPFC were affected by rTMS, whereas with related word pairs the engagement of DLPFC was not so conspicuous to be influenced by rTMS. As shown in Figure 2, the performance was different within blocks, $F(1,11)=6.81, p<.05$, as indicated also by the interaction between category and block, $F(5,55)=$ $2.70, p<.05$. Directed post hoc comparison (Wilcoxon 
Figure 1. Task schematics. Subjects fixated a small cross at the center of the screen and monitored for the appearance of target stimuli that consisted of word pairs presented on the monitor for $2000 \mathrm{msec}$. Trains of rTMS $(20 \mathrm{~Hz}, 10 \%$ below individual motor threshold) were delivered in the first $500 \mathrm{msec}$, simultaneously with the word pair presentation to the left or right DLPFC, when required by the experimental design. Participants had to identify related or unrelated word pairs in the encoding phase. One hour later, in the retrieval phase, they had to recognize words previously presented (tests) among new stimuli (distractors).
ENCODING PHASE

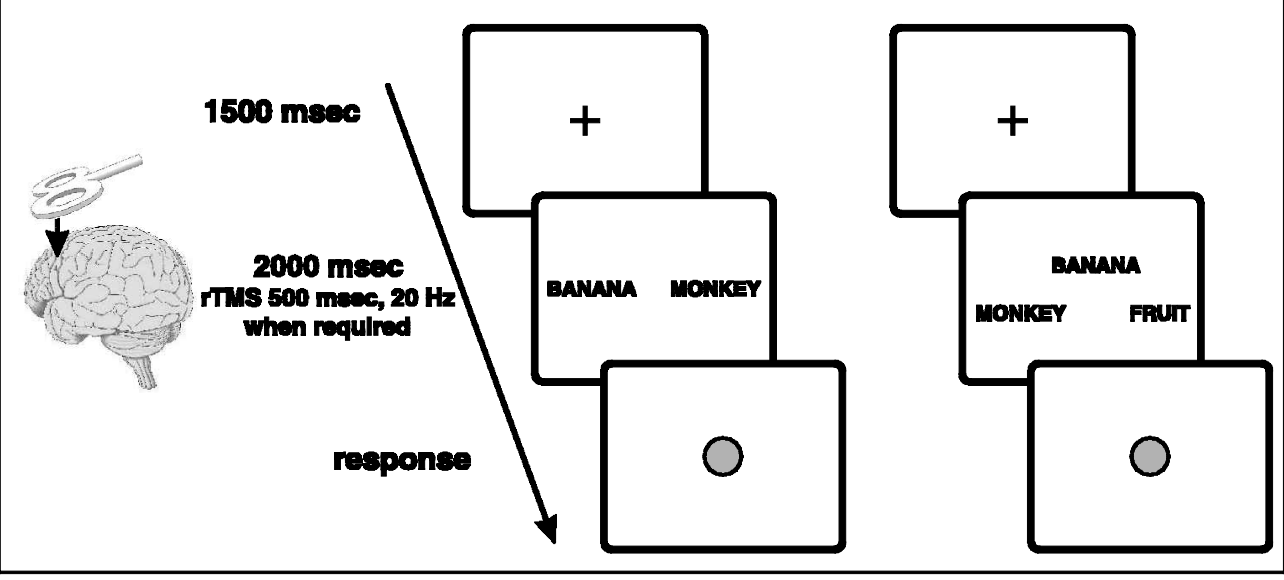

signed-rank test) showed that, indeed, performance with real rTMS was different from performance with "sham" rTMS or baseline. Specifically, for the unrelated word pairs the conditions L-Enc, R-Enc and R-Ret differed significantly from sham, baseline, and L-Ret $(p<$ .05 ), suggesting a bilateral engagement of the DLPFC in encoding and a right engagement in retrieval. None of the other comparisons were significant.

Reaction times for each response were consistently faster in encoding than in retrieval, as expected from the different cognitive demand, in agreement with the previous study (Rossi et al., 2001). After using logistic regressions to verify that no significant interaction was present between RTs and error rate, a further ANOVA on RTs was carried out with category and block as variables. The only significant association was the shortening of RTs for related, with respect to unrelated words, both in encoding, $F(1,95)=18.43, p<.05$, and in retrieval, $F(1,95)=49.54, p<.05$.

\section{DISCUSSION}

The present findings are consistent with the results of previous TMS studies (Epstein, Sekino, Yamaguchi, Kamiya, \& Ueno, 2002; Rossi et al., 2001) indicating the functional role of DLPFC in long-term episodic memory encoding and retrieval.

Whereas in the previous study using pictorial material, in agreement with the HERA model, a significant left hemispheric effect was present for encoding, in the current experiment rTMS to both right and left DLPFC had a significant interference effect. This finding suggests that in the case of verbal material, both DLPFCs play a key role in encoding mechanisms. Furthermore, the effect was specific for semantically unrelated word pairs, therefore suggesting a specific role of DLPFC(s) when novel information must be memorized.
These findings in encoding were unexpected. Based on neuropsychological evidence, an asymmetry, with left hemisphere predominance, could be expected for verbal material. It is noteworthy that a bilateral DLPFC activation has also been reported in some other experiments with verbal encoding, using word pairs with high imagery content (Schmidt et al., 2002; Mottaghy et al., 1999), whereas during encoding of word pairs with low

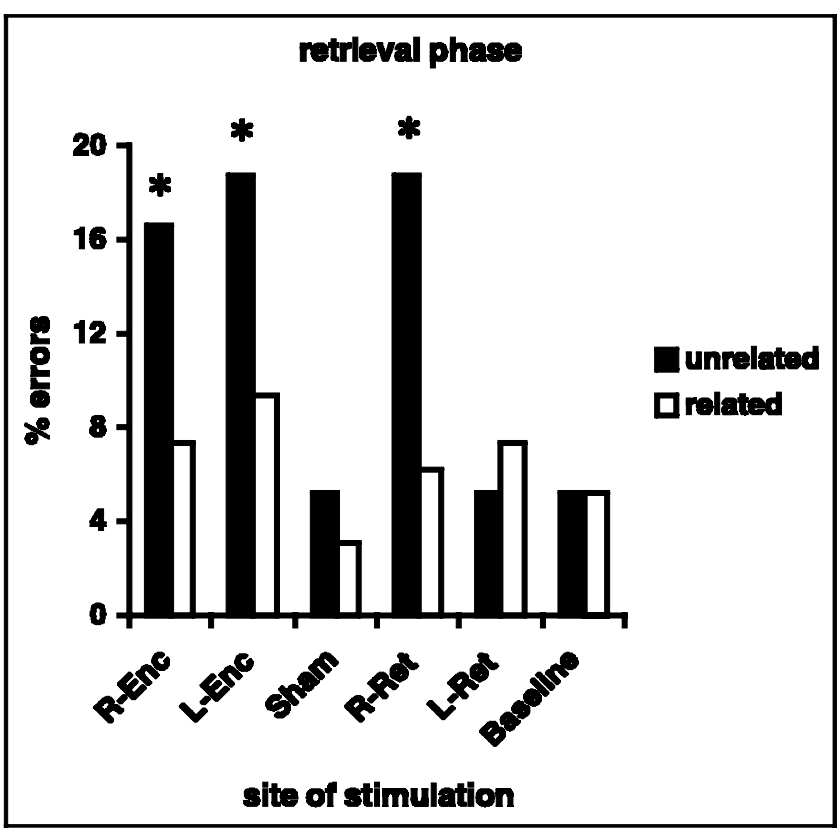

Figure 2. Behavioral results. The graph shows the percentage of errors to detect target stimuli in the retrieval phase for the six conditions divided by type of material. The performance is significantly worst for the unrelated word pairs. In particular, the error rate is higher in the following blocks: R-Enc, L-Enc, and R-Ret, suggesting a direct involvement of DLPFC in these blocks. In the other blocks the error rate is similar. ${ }^{*} p<.05$. 
imagery content, activity was present in the left DLPFC (Schmidt et al., 2002). A bilateral DLPFC activation in the encoding phase was also reported in other studies (Lee, Robbins, Pickard, \& Owen, 2000; Opitz, Mecklinger, \& Friederici, 2000).

It may be speculated that during the present task the participants had to perform a deep manipulation, in agreement with the predictions of the so called "dualcoding theory." This theory claims that processing of abstract nouns relies on verbal code representations of the left hemisphere only, whereas concrete nouns additionally access a second image-based processing system in the right hemisphere (Paivio, 1986). The involvement of the right DLPFC during encoding of word pairs of high imagery content could parallel this strategy. Therefore, bilateral DLPFC involvement may depend on a mixture of verbal as well as nonverbal strategies in the context of episodic encoding.

The results for retrieval are in agreement with the predictions. Many studies on verbal episodic retrieval reported activation in the right rather then in the left DLPFC (Allan, Dolan, Fletcher, \& Rugg, 2000; Saykin et al., 1999; Wagner et al., 1998; Cabeza et al., 1997; Fletcher, Shallice, Frith, Frackowiak, \& Dolan, 1996; Rugg et al., 1996; Kapur et al., 1995; Shallice et al., 1994; Tulving et al., 1994; Squire et al., 1992). These data together with our results for verbal recognition are consistent with the HERA generalization. However, bilateral effects during episodic retrieval have been also found (Schmidt et al., 2002; Henson, Shallice, \& Dolan, 1999; Henson, Rugg, Shallice, \& Dolan, 2000; Halsband et al., 1998; Rugg, Fletcher, Frith, Frackowiak, \& Dolan, 1997; Schacter, Buckner, Koutstaal, Dale, \& Rosen, 1997).

According to Nolde, Johnson, and D'Esposito (1998) and Nolde, Johnson, and Raye (1998), whether left prefrontal activation is or is not observed is systematically related to the "reflective demands" of the retrieval tasks. When these demands are low, as in forced-choice recognition, only the right prefrontal cortex is engaged. When reflective demands are high, however, as is true of tasks requiring the evaluation of episodic detail, the left prefrontal cortex is also engaged.

The reduced recognition of new associations after right stimulation during retrieval suggests that the rTMS-induced disrupting effect is direct, as it takes place immediately after the stimulation period, whereas retrieval effort is active. This also points to a selective specialization of the right DLPFC in the monitoring phase of retrieval (Henson et al., 1999; Shallice et al., 1994) during a recognition task of word pairs. This monitoring function may be particularly crucial during recognition performance as subjects must continuously monitor information in long-term store and compare it during the test phase to determine the correct response.

In conclusion, the present findings extend our previous results on the role of the DLPFC in episodic memory to the verbal domain and confirm the crucial role of the right DLPFC in retrieval mechanisms, independently of the nature (verbal or visuospatial) of the stimuli. In agreement with other neuroimaging results, they indicate that the encoding asymmetry is affected in complex ways by the nature of the material to be remembered. Finally, they indicate that the contribution of the DLPFC appears to be specific for novel material.

\section{METHODS}

\section{Participants}

Twelve healthy volunteers ( 4 males and 8 females), aged between 20 and 34 years (mean age $=27$ ) gave their written informed consent before the experiment. They were right-handed (mean score on the Edinburgh Handedness Inventory $=85.2 \%$ ) and had normal or corrected-to-normal vision. All participants were native Italian speakers.

\section{Experimental Task}

Study words were Italian nouns (from two to four syllables) that were between four and nine letters and of moderate frequency (De Mauro, Mancini, Vedovelli, \& Voghera, 1993). Word pairs varied in terms of imagery according to a Italian linguistic database (Barca, Burani, \& Arduino, 2001) of 626 nouns on a scale between 1 and 7, 1 being of low and 7 being of high imagery content. Words with an imagery rating $>5$ were defined as imaginable. For our study, we used words in the range between 5 and 7 (mean 5.95).

The experiment included six blocks in the encoding phase followed by six blocks of retrieval, which were presented in a counterbalanced order. For each block of the encoding phase, 16 word pairs ( 8 semantically related and 8 unrelated) were randomly presented on the monitor for $2000 \mathrm{msec}$, with two intertrial intervals (7000-8000 msec). Pair words were preceded by a fixation point for $1500 \mathrm{msec}$. Subjects had to judge whether the words of a pair were semantically related or not.

Participants were instructed to press one of the two buttons (e.g., left, if the word pairs were semantically related; right, if the word pairs were unrelated) as quickly as possible after the presentation of a green circle in the middle of the screen ("go" signal).

The six encoding blocks had six corresponding retrieval blocks ( $1 \mathrm{hr}$ later). In the retrieval blocks the first word of each pair was again presented among the words presented in the encoding phase (target) and a novel word (distractor). All the events in the retrieval phase had the same timing as the encoding event. Subjects were instructed to press the left or right key according to the position of the word that had been seen previously (see Figure 1). The correct responses during encoding and retrieval phases were evenly distributed across left and right button presses (eight each). 
The six encoding/retrieval blocks were labeled according to the type (active or sham) and the side (left/right) of the rTMS applied on the DLPFCs. These were: R-Enc (= right rTMS in encoding, no stimulation in retrieval); L-Enc (= left rTMS in encoding, no stimulation in retrieval); sham (= left rTMS in encoding and right in retrieval); R-Ret (= no stimulation in encoding and right rTMS in retrieval); L-Ret (= no stimulation in encoding and left rTMS in retrieval); baseline, which served as a reference condition, consisted of no stimulation in encoding or retrieval. Differences in error rate were assessed by repeated-measures analysis of variance (ANOVA), using the Huynh-Feldt epsilon correction where appropriate. The factors tested were "category" (related word pairs vs. unrelated word pairs) and "block" (R-Enc, L-Enc, sham, R-Ret, L-Ret, and baseline).

A total of 288 words were used. For each cue word we chose two different words.

For the semantically related pairs, the word association norms were drawn from the DPSS psycholinguistics database (Peressotti, Pesciarelli, \& Job, 2002). Because the test phase was a recognition task, we chose stimuli with a degree of association between 15\% and 30\% in order to match the degree of association for test and distractor words. These norms provide the information that, for example, $26.66 \%$ of people give "onion" as the first word they think of in response to the word "garlic." With regard to the semantically unrelated word pairs, they were chosen on the basis of belonging to distant semantic categories and of lack of association according to the same norms.

All stimuli were generated using Superlab software with an RB-400 response pad running on an IBM 300GL personal computer with a 17 -in. monitor.

\section{Application of rTMS}

Subjects were seated comfortably in a reclining chair at a distance of $85 \mathrm{~cm}$ from the screen. rTMS was delivered using a Magstim Super Rapid(III) magnetic stimulator with a figure-of-eight (double wings of $70-\mathrm{mm}$ diameter) coil, which induces a maximum magnetic field of $2.2 \mathrm{~T}$ at the scalp site. The subjects wore a Lycra cap on which the positions of all the electrodes from the International 10/20 EEG system were reproduced. Individual resting excitability thresholds of stimulation were previously determined for right and left motor cortices by standard procedure (Rossini et al., 1994). The intensity of stimulation was then reduced by $10 \%$. Thus, left and right DLPFC were stimulated, when required, at an intensity that was subthreshold for any stimulation spread to motor cortex as well as to avoid blink reflex, which might otherwise interfere with motor performance or reduce recognition.

Left and right DLPFCs were stimulated by placing the anterior end of the junction of the two coil wings on F3 and F4, respectively. F3 and F4 locations on the subject's scalp were automatically identified using SofTaxic Navigator system, from digitized skull landmarks (nasion, inion, and two preauricolar points) and about 40 scalp points (Fastrak Polhemus digitizer). Although individual radiological head images (i.e., magnetic resonance images [MRIs]) were not available, Talairach coordinates of cortical sites underlying $\mathrm{F} 3$ and $\mathrm{F} 4$ locations were automatically estimated by the SofTaxic Navigator from an MRI-constructed stereotaxic template (accuracy of $\pm 1 \mathrm{~cm}$, Talairach space). Talairach coordinates of cortical sites underlying $\mathrm{F} 3$ and $\mathrm{F} 4$ were corresponding to Brodmann's area 9 of the left and right hemispheres (Talairach \& Tournoux, 1988).

Trains of $10 \%$ subthreshold rTMS $(500 \mathrm{msec}, 20 \mathrm{~Hz})$ were delivered-when required by the experimental design-simultaneously to word presentation. The same intensity and timing of rTMS was used for sham stimulation: Here, the coil was still centered on F3 and F4 but was held perpendicular to the scalp surface. By adopting this procedure no magnetic stimulation reached the brain during the sham condition, whereas subjective sensations of coil-scalp contact and discharge noise were similar to the real simulation. The institutional Bioethics Committee approved the protocol.

\section{Acknowledgments}

This research was supported by a grant provided by the Italian Ministry of Health. We thank Katiuscia Sosta for experimental help.

Reprint requests should be sent to Carlo Miniussi, Neurophysiology and Neuropsychology Laboratory IRCCS "San Giovanni di Dio-FBF," Via Pilastroni, 425125 Brescia, Italy, or via e-mail: c.miniussi@oh-fbf.it.

\section{REFERENCES}

Allan, K., Dolan, R. J., Fletcher, P. C., \& Rugg, M. D. (2000). The role of the right anterior prefrontal cortex in episodic retrieval. Neuroimage, 11, 217-227.

Barca, L., Burani, C., \& Arduino, L. S. (2001). Word naming times and norms for age of acquisition, frequency, familiarity, imageability, concreteness, and other variables for Italian nouns. Giornale Italiano di Psicologia, 4 , $839-854$.

Brewer, J. B., Zhao, Z., Desmond, J. E., Glover, G. H., \& Gabrieli, J. D. E. (1998). Making memories: Brain activity that predicts whether visual experiences will be remembered or forgotten. Science, 281, 1185-1187.

Cabeza, R., Mangels, J., Nyberg, L., Habib, R., Houle, S., McIntosh, A. R., \& Tulving, E. (1997). Brain regions differentially involved in remembering what and when: A PET study. Neuron, 19, 863-870.

Cabeza, R., \& Nyberg, L. (2000). Imaging cognition: II. An empirical review of 275 PET and fMRI studies. Journal of Cognitive Neuroscience, 12, 1-47.

De Mauro, T., Mancini, F., Vedovelli, M., \& Voghera, M. (1993). Lessico di frequenza dell'italiano parlato. Milano: ETASLIBRI.

Epstein, C. M., Sekino, M., Yamaguchi, K., Kamiya, S., \& Ueno, S. (2002). Asymmetries of prefrontal cortex in 
human episodic memory: Effects of transcranial magnetic stimulation on learning abstract patterns. Neuroscience Letters, 320, 5-8.

Fletcher, P. C., \& Henson, R. N. A. (2001). Frontal lobes and human memory. Insights from functional neuroimaging. Brain, 124, 849-881.

Fletcher, P. C., Shallice, T., Frith, C. D., Frackowiak, R. S., \& Dolan, R. J. (1996). Brain activity during memory retrieval. The influence of imagery and semantic cueing. Brain, 119, $1587-1596$.

Goldman-Rakic, P. S. (1996). The prefrontal landscape: Implications of functional architecture for understanding human mentation and central executive. Philosophical Transactions of the Royal Society of London, Series B. Biological Sciences, 351, 1445-1453.

Gorno-Tempini, M. L., Price, C. J., Josephs, O., Vandenberghe, R., Cappa, S. F., Kapur, N., Frackowiak, R. S., \& Tempini, M. L. (1998). The neural systems sustaining face and proper-name processing. Brain, 121, 2103-2118.

Halsband, U., Krause, B. J., Schmidt, D., Herzog, H., Tellmann, L., \& Muller-Gartner, H. W. (1998). Encoding and retrieval in declarative learning: A positron emission tomography study. Behavioural Brain Research, 97, 69-78.

Henson, R. N. A., Rugg, M. D., Shallice, T., \& Dolan, R. J. (2000). Confidence in recognition memory for words: Dissociating right prefrontal roles in episodic retrieval. Journal of Cognitive Neuroscience, 12, 913-923.

Henson, R. N. A., Shallice, T., \& Dolan, R. J. (1999). Right prefrontal cortex and episodic memory retrieval: A functional MRI test of the monitoring hypothesis. Brain, 122, $1367-1381$.

Kapur, S., Craik, F., Jones, C., Brown, G. M., Houle, S., \& Tulving, E. (1995). Functional role of the prefrontal cortex in retrieval of memories: A PET study. NeuroReport, 6, $1880-1894$.

Kelley, W. M., Miezin, F. M., McDermott, K. B., Buckner, R. L., Raichle, M. E., Cohen, N. J., Ollinger, J. M., Akbudak, E., Conturo, T. E., Snyder, A. Z., \& Petersen, S. E. (1998). Hemispheric specialization in human dorsal frontal cortex and medial-temporal lobe for verbal and nonverbal memory encoding. Neuron, 20, 927-936.

Lee, A. C. H., Robbins, T. W., Pickard, J. D., \& Owen, A. M. (2000). Asymmetric frontal activation during episodic memory: The effects of stimulus type on encoding and retrieval. Neuropsychologia, 38, 677-692.

Martin, A., Haxby, J. V., Lalonde, F. M., Wiggs, C. L., \& Ungerleider, L. G. (1995). Discrete cortical regions associated with knowledge of colour and knowledge of action. Science, 270, 102-105.

Martin, A., Wiggs, C. L., Ungerleider, L. G., \& Haxby, J. V. (1996). Neural correlates of category-specific knowledge. Nature, 379, 649-652.

Mottaghy, F. M., Shah, N. J., Krause, B. J., Schmidt, D., Halsband, U., Jäncke, L., \& Müller-Gärtner, H.-W. (1999). Neuronal correlates of encoding and retrieval in episodic memory during a paired-word association learning task: A functional magnetic resonance imaging study. Experimental Brain Research, 128, 332-342.

Nolde, S. F., Johnson, M. K., \& D'Esposito, M. (1998). Left prefrontal activation during episodic remembering: An event-related fMRI study. NeuroReport, 9, 3509-3514.

Nolde, S. F., Johnson, M. K., \& Raye, C. L. (1998). The role of the prefrontal cortex during tests of episodic memory. Trends in Cognitive Science, 2, 399-406.

Nyberg, L., Cabeza, R., \& Tulving, E. (1996). PET studies of encoding and retrieval: The HERA model. Psychonomic Bulletin and Review, 3, 135-148.

Nyberg, L., Tulving, E., Habib, R., Nilson, L. G., Kapur, S.,
Houle, S., Cabeza, R., \& McIntosh, A. R. (1995). Functional brain maps of retrieval mode and recovery of episodic information. NeuroReport, 7, 249-252.

Opitz, B., Mecklinger, A., \& Friederici, A. D. (2000). Functional asymmetry of human prefrontal cortex: Encoding and retrieval of verbally and nonverbally coded information. Learning and Memory, 7, 85-96.

Paivio, A. (1986). Mental representations: A dual coding theory. Oxford: University Press.

Peressotti, F., Pesciarelli, F., \& Job, R. (2002). Le associazioni verbali PD/DPSS: Norme per 294 parole. Giornale Italiano di Psicologia, 29, 153-170.

Petersen, S. E., van Mier, H., Fiez, J. A., \& Raichle, M. E. (1998). The effects of practice on the functional anatomy of task performance. Proceedings of the National Academy of Sciences, U.S.A., 95, 853-860.

Petrides, M. (1994). Frontal lobes and working memory: Evidence from investigation of the effects of cortical excisions in nonhumans primates. In F. Boller \& J. Grafmann (Eds.), Handbook of Neuropsychology. Amsterdam: Elsevier.

Price, C. J., \& Friston, K. J. (1999). Scanning patients with tasks they can perform. Human Brain Mapping, 8, 102-108.

Roland, P. E., \& Gulyas, B. (1995). Visual memory, visual imagery, and visual recognition of large field patterns by the human brain: Functional anatomy by positron emission tomography. Cerebral Cortex, 5, 79-93.

Rossi, S., Cappa, S. F., Babiloni, C., Pasqualetti, P., Miniussi, C., Carducci, F., Babiloni, F., \& Rossini, P. M. (2001). Prefrontal cortex in long-term memory: An "interference" approach using magnetic stimulation. Nature Neuroscience, 9, 948-952.

Rossini, P. M., Barker, A. T., Berardelli, A., Caramia, M. D., Caruso, G., Cracco, R. Q., Dimitrijevic, M. R., Hallett, M., Katayama, Y., Lücking, C. H., Maertens de Noordhout, A., Marsden, C., Murray, N., Rothwell, J. C., Swash, M., \& Thomberg, C. (1994) Non-invasive electrical and magnetic stimulation of the brain, spinal cord and roots: Basic principles and procedures for routine clinical application. Report of an IFCN committee. Electroencephalography and Clinical Neurophysiology, 91, 79-92.

Rugg, M. D., Fletcher, P. C., Frith, C. D., Frackowiak, R. S., \& Dolan, R. J. (1996). Differential activation of the prefrontal cortex in successful and unsuccessful memory retrieval. Brain, 119, 2073-2083.

Rugg, M. D., Fletcher, P. C., Frith, C. D., Frackowiak, R. S., \& Dolan, R. J. (1997). Brain regions supporting intentional and incidental memory: A PET study. NeuroReport, 8 , $262-273$.

Rugg, M. D., \& Wilding, E. L. (2000). Retrieval processing and episodic memory. Trends in Cognitive Science, 4, 108-115.

Saykin, A. J., Johnson, S. C., Flashman, L. A., McAllister, T. W., Sparling, M., Darcey, T. M., Moritz, C. H., Guerin, S. J., Weaver, J., \& Mamourian, A. (1999). Functional differentiation of medial temporal and frontal regions involved in processing novel and familiar words: An fMRI study. Brain, 122, $1963-1971$.

Schacter, D. L., Alpert, N. M., Savage, C. R., Rauch, S. L., \& Albert, M. S. (1996). Conscious recollection and the human hippocampal formation: Evidence from positron emission tomography. Proceedings of the National Academy of Sciences, U.S.A., 93, 321-325.

Schacter, D. L., Buckner, R. L., Koutstaal, W., Dale, A. M., \& Rosen, B. R. (1997). Late onset of anterior prefrontal activity during true and false recognition: An event-related fMRI study. Neuroimage, 6, 259-269.

Schmidt, D., Krause, B. J., Mottaghy, F. M., Halsband, U., Herzog, H., Tellmann, L., \& Müller-Gärtner, H. W. (2002). Brain systems engaged in encoding and retrieval of 
word-pair associates independent of their imagery content or presentation modalities. Neuropsychologia, 40, 457-470.

Shallice, T., Fletcher, P., Frith, C. D., Grasby, P., Frackowiak, R. S., \& Dolan, R. J. (1994). Brain regions associated with acquisition and retrieval of verbal episodic memory. Nature, 368, 633-635.

SofTaxic Navigator-Stereotaxic Navigator System. E. M. S. s.r.l., Italy. www.emsmedical.net.

Squire, L. R., Ojemann, J. G., Miezin, F. M., Petersen, S. E., Videen, T. O., \& Raichle, M. E. (1992). Activation of the hippocampus in normal humans: A functional anatomical study of memory. Proceedings of the National Academy of Sciences, U.S.A., 89, 1837-1841.

Stuss, D. T., Eskes, G. A., \& Foster, J. K. (1994). Experimental neuropsychological studies of frontal lobe functions. In F. Boller \& J. Grafman (Eds.), Handbook of neuropsychology. Amsterdam: Elsevier.

Talairach, J., \& Tournoux, P. (1988). Coplanar stereotaxic Atlas of the Human Brain. Stuggart, Germany: Thieme.

Thompson-Schill, S. L., D'Esposito, M., Aguirre, G. K., \&
Farah, M. J. (1997). Role of the left inferior prefrontal cortex in retrieval of semantic knowledge: A re-evaluation. Proceedings of the National Academy of Sciences, U.S.A., 94, $14792-14797$.

Tulving, E., Kapur, S., Craik, F. I. M., Moscovitch, M., \& Houle, S. (1994). Hemispheric encoding/retrieval asymmetry in episodic memory: Positron emission tomography findings. Proceedings of the National Academy of Sciences, U.S.A., 91, 2016-2020.

Vandenberghe, R., Price, C., Wise, R., Josephs, O., \& Frackowiak, R. S. (1996). Functional anatomy of a common semantic system for words and pictures. Nature, 383, 254-256.

Wagner, A. D., Poldrack, R. A., Eldridge, L. L., Desmond, J. E., Glover, G. H., \& Gabrieli, J. D. (1998). Material-specific lateralization of prefrontal activation during episodic encoding and retrieval. NeuroReport, 9, 3711-3717.

Walsh, V., \& Cowey, A. (2000). Transcranial magnetic stimulation and cognitive neuroscience. Nature Reviews Neuroscience, 1, 73-79. 